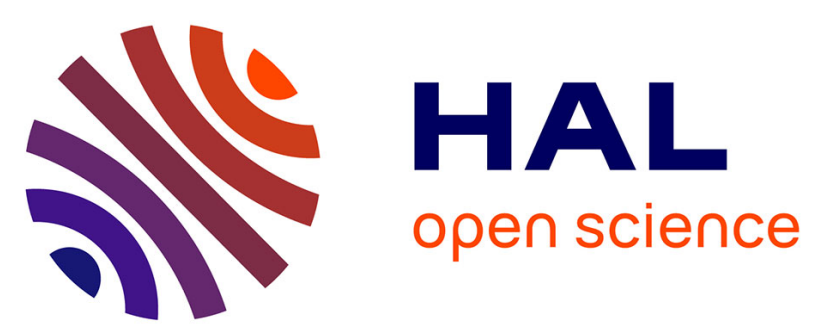

\title{
Master Role of Glucose-6 Phosphate in Cell Signalling and Consequences of its Deregulation in the Liver and Kidneys
}

Gilles Mithieux, Fabienne Rajas

\section{- To cite this version:}

Gilles Mithieux, Fabienne Rajas. Master Role of Glucose-6 Phosphate in Cell Signalling and Consequences of its Deregulation in the Liver and Kidneys: Key metabolic role of glucose-6 phos-

phate. Vinood Patel. Molecular Nutrition: Carbohydrates, Chapter 10, Elsevier, pp.173-189, 2019, 9780128498866. 10.1016/B978-0-12-849886-6.00021-5 . inserm-02383971

HAL Id: inserm-02383971

https://www.hal.inserm.fr/inserm-02383971

Submitted on 28 Nov 2019

HAL is a multi-disciplinary open access archive for the deposit and dissemination of scientific research documents, whether they are published or not. The documents may come from teaching and research institutions in France or abroad, or from public or private research centers.
L'archive ouverte pluridisciplinaire HAL, est destinée au dépôt et à la diffusion de documents scientifiques de niveau recherche, publiés ou non, émanant des établissements d'enseignement et de recherche français ou étrangers, des laboratoires publics ou privés. 


\section{Master Role of Glucose-6 Phosphate in Cell Signalling and Consequences of its \\ Deregulation in the Liver and Kidneys}

Fabienne Rajas[1] and Gilles Mithieux

Institut National de la Santé et de la Recherche Médicale, U1213, Lyon, F-69008, France

Université de Lyon, Lyon, F-69008 France

Université Lyon I, Villeurbanne, F-69622 France

[1] Corresponding author: Dr. Fabienne Rajas, Ph.D.

Inserm U1213, Université Lyon 1 Laennec

7 rue Guillaume Paradin, 69372 Lyon cedex 08 France

Tel: +33478771028 / Fax: +33478778762

E-mail: fabienne.rajas@univ.lyon1.fr

Second author: Dr. Gilles Mithieux, Ph.D.

Inserm U1213, Université Lyon 1 Laennec

7 rue Guillaume Paradin, 69372 Lyon cedex 08 France

Tel: +33478778788 / Fax: +33478778762

E-mail: gilles.mithieux@univ.lyon1.fr

\section{Word count}

5822 words (abstract, key words and references not included in word count)

4 Figures

79 References

2572 words in references

Running title: Key metabolic role of glucose- 6 phosphate 


\section{Summary:}

Maintaining blood glucose levels through periods of fasting or excess of nutrients is a crucial function to the survival of the organism. In this review, we describe the glucose metabolism during the fed to fasted transition and in two metabolic diseases characterized by either an overproduction of glucose and hyperglycemia, i.e. type 2 diabetes or by a deficiency of glucose production and hypoglycemia, i.e. Glycogen Storage Disease Type I. In these two pathologies, glucose- 6 phosphate plays a master role in cell signaling. Indeed, the increased flux downstream of glucose- 6 phosphate leads to a complete metabolism reprograming and an accumulation of lipids in the liver and kidneys. Thus, with time, type 2 diabetic patients and GSDI patients can develop hepatic tumors and chronic kidney disease.

Key words: Carbohydrate response element binding protein, ChREBP; Chronic kidney disease; Diabetes; Fasting; Glycogen storage disease type I; Hepatocellular adenoma and carcinoma; Lipids; Non alcoholic fatty liver disease, Steatosis.

\section{List of abbreviations:}

ACE: Converting enzyme; ChREBP: carbohydrate response element binding protein; CKD: chronic kidney disease; G6P: glucose-6 phosphate; G6Pase: glucose-6 phosphatase (G6Pase); GNG: Gluconeogenesis; GCK: Glucokinase; GSDI: glycogen storage disease type I; HCA: hepatocellular adenoma; HCC: hepatocellular carcinoma; NAFLD: non-alcoholic fatty liver diseases; PKC: protein kinase C; PPP: pentose phosphate pathway; SITR2: Sirtuin 2; TG: triglycerides; TGF- $\beta 1$ : transforming growth factor- $\beta 1$; VLDL: very low density lipoproteins (VLDL). 
Maintaining normoglycemia is a finely tuned, crucial physiological function. Indeed, most mammals, including humans, are incapable of tolerating hypoglycemia for more than a few minutes. Glucose is the major source of energy for most cells and provides precursors for biomolecule synthesis, such as nucleotides. Once a glucose molecule has passed from the bloodstream into a cell, it is rapidly phosphorylated into glucose-6 phosphate (G6P) and enters the glycolysis pathway (Figure 1). In addition, G6P enters the pentose phosphate pathway (PPP), where it is oxidized by glucose- 6 phosphate dehydrogenase to produce ribose 5 -phosphate, an intermediate metabolite in nucleotide synthesis required for DNA replication, as well as the production of the biological reductant NADPH. Glucose can also be stored as glycogen in the liver and skeletal muscle. When the storage capacity of glucose under the form of glycogen is exceeded, G6P is transformed into fat via de novo lipogenesis pathway. While hepatic lipid synthesis is a normal physiological process, excessive storage of lipids in the liver leads to the development of steatosis that represents a pre-pathological state possibly evolving in nonalcoholic fatty liver diseases (NAFLD). Thus, G6P is situated at the crossroad of several metabolic pathways and thus plays a key role in cell metabolism. In this review, we will focus on G6P metabolism in the liver and kidneys, the two major glucose-producing organs. We will also analyze the consequences of its deregulation in two metabolic diseases: type 2 diabetes and glycogen storage disease type I (GSDI). Indeed, abnormal plasma concentrations of glucose result in deleterious effects that can alter the normal functioning of the whole organism. Decreased glucose levels are of particular danger for several tissues, especially in neuronal cells or erythrocytes that require glucose ceaselessly under normal physiological conditions. Thus, hypoglycemia can lead to impaired brain function and in more extreme situations it can lead to death. Conversely, hyperglycemia results in non-enzymatic glycosylation (glycation) and thus loss-of-function of proteins, glucose-induced oxidative damage and other adverse effects such as macrovascular and microvascular complications. While type 2 diabetes is characterized by an overproduction of glucose and hyperglycemia, GSDI is due to an absence of glucose production 
leading to severe hypoglycemia between two meals. Thus, these two pathologies could be considered as "mirror" diseases. However, they share similar hepatic and renal metabolic perturbations. Therefore, type 2 diabetic patients and GSDI patients are both prone to hepatic steatosis and tumor development and to chronic kidney disease (CKD) that appear with time (Figure 2).

\section{Maintenance of blood glucose}

\section{Summary points}

- This chapter focuses on metabolic adaptions during the fed-to-fasted period to maintain blood glucose.

- In the fed state, glucose is stored under the form of glycogen in the liver and muscles.

- During the post-prandial period, glucose is mainly produced from glycogen degradation (glycogenolysis) in the liver, to maintain glycemia.

- Hepatic glycogen metabolism is controlled by the coordinated action of two enzymes, glycogen synthase and glycogen phosphorylase, both of which are regulated by phosphorylation (regulated by pancreatic insulin or glucagon) and allosteric modulators such as glucose and glucose- 6 phosphate.

- During long-term food deprivation, glucose is produced by gluconeogenesis in the liver, kidney and intestine.

- Gluconeogenesis is the synthesis of glucose from non-sugar carbon compounds. The main precursors are lactate and alanine in the case of liver gluconeogenesis, whereas glutamine is an essential substrate for gluconeogenesis in the kidneys and intestine.

- Glucose homeostasis is balanced by nutrient sensing and hormones that control tissuespecific rates of glucose utilization and production. Insulin induces glucose utilization by tissues and inhibits glucose production, while glucagon activates glucose production. 
The maintenance of blood glucose between 0.7 and $1.2 \mathrm{mg} / \mathrm{dl}(4-7 \mathrm{mmol} / \mathrm{l})$ is a critical requirement for the body to maintain metabolism and function of several organs including the brain, kidneys or intestine. While glucose is generally considered as a major source of energy, ketone bodies represent the major energy source of several organs, including the brain, during fasting periods. Thus, during fasting, body reduces glucose oxidation to supply PPP for the synthesis of nucleotides, which is essential for cell proliferation (Soty et al., 2017). Thereby, global glucose turnover decreases and glucose is preserved to supply 3-carbon skeletons to the body during fasting.

During the fed-to-fasted transition, glucose metabolism is finely tuned by a multi-organ crosstalk involving hormonal regulation and both central and splanchnic nervous systems (Donovan and Watts, 2014). Insulin and glucagon that are produced by $\beta$ and $\alpha$ cells, respectively, in the endocrine pancreas, are the main regulators of blood glucose levels. In addition to insulin and glucagon, counter-regulatory hormones such as epinephrine are also involved in this tight regulation. The liver and kidneys play a central role in glucose homeostasis by coordinating the storage/utilization and the synthesis of glucose between two meals and fasting in response to hormonal signaling. Moreover, reabsorption of filtered glucose in the renal proximal tubules is also an essential process to prevent excretion of glucose in the urine.

In the fed state, the presence of high insulin level favors tissue glucose utilization through the glycolytic pathway and storage of glucose in glycogen in the liver and muscles (around 30$40 \%$ of alimentary glucose). Glycogen is a polymer composed of glucose molecules forming long linear chains linked by $\alpha(1-4)$ bonds and fixed on main ramification chains by $\alpha(1-6)$ branching bonds (Roach et al., 2012). At a molecular level, glucose inhibits glycogen phosphorylase phosphatase, preventing glycogen degradation, whereas G6P allosterically activates glycogen synthase, thus favoring glycogen synthesis (Agius, 2015). Hepatic glycogen represents around 5 $\%$ of the weight of the liver. A deregulation of its storage and/or metabolism in the liver and/or muscle is responsible for glycogen storage diseases (GSD), which are a family of rare genetic diseases (Hicks et al., 2011; Weinstein et al., 2018). Hypoglycemia is the primary manifestation 
of the hepatic GSDs (types 0,I, III, VI IX and XI). Contrarily to the liver and muscle, glycogen is not accumulated in the normal kidneys. However, when G6P is present in excessive concentrations, this metabolite can be stored as glycogen in the kidneys, as observed in GSDI and Fanconi-Bickel syndrome (due to GLUT 2 mutations), leading to nephromegaly (Berry et al., 1995; Martens et al., 2009). In diabetes, renal glycogen accumulation was reported in hyperglycemic conditions, but further studies are necessary to better understand the tubular formation of glycogen deposits and their relevance in the diabetic kidney (Vallon and Komers, 2011).

In the fasted state, the decrease in glycemia reduces intracellular glucose and G6P levels, activating glycogen degradation. Glycogen stores are considered to contribute to endogenous glucose production for about 10-12h in rodents and 20h in human subjects (Mutel et al., 2011; Soty et al., 2017). During this post-absorptive period, the liver accounts for at least $80 \%$ of glucose production, mainly ensured by glycogenolysis. The stimulation of hepatic glycogenolysis during fasting or adrenergic stimulation is dependent on cAMP-PKA signaling. Indeed, both glycogen synthase and glycogen phosphorylase are phosphorylated by PKA, which results in a coordinated inhibition of glycogen synthase and activation of glycogen phosphorylase. Furthermore, the increase in glucagon activates the gluconeogenic pathway by inducing the expression of phosphoenolpyruvate carboxykinase-cytosolic form (encoded by Pck1) and glucose-6 phosphatase (G6Pase). Gluconeogenesis (GNG) is the de novo synthesis of glucose from non-carbohydrate substrates that takes place only in the liver, kidney and intestine (Soty et al., 2017). G6Pase catalyzes the hydrolysis of G6P into glucose and inorganic phosphate, leading to the production of glucose in the bloodstream. This enzyme is expressed exclusively in gluconeogenic organs, but not in the muscles. Therefore, G6P is not converted into glucose in the muscle and thus not released in the bloodstream for glucose maintenance, but it is used solely in glycolysis to produce energy for muscle contraction. While the contribution of hepatic glucose production decreases during fasting, the contribution of renal GNG is increased to represent more than 50\% of glucose production in fasting rats or humans (Gerich et al., 2001; Owen et al., 1969; Pillot et al., 2009). Moreover, we showed that intestinal GNG takes place to maintain 
glycemia during prolonged fasts (Croset et al., 2001; Penhoat et al., 2014). It is noteworthy that glucose produced by the gut exerts in the post-absorptive state beneficial metabolic effects. By delivering glucose directly in the portal vein, where glucose is sensed by the nervous system, it induces a gut-brain glucose signal (Soty et al., 2017). This portal glucose signal positively controls different metabolic functions, such as food intake and insulin sensitivity. Thus, the regulation of endogenous glucose production is finely regulated in the three gluconeogenic organs, allowing them to coordinately maintain blood glucose. While glucagon is mainly known to modulate hepatic glucose production (Jiang and Zhang, 2003), it has also been shown to modulate the transcription of $G 6 p c$ in the kidneys and intestine, thus modulating renal and intestinal glucose production (Mutel et al., 2011). Recently, we have demonstrated a possible involvement of a crosstalk between the kidneys and intestine, via the vitamin D system, and the intestine and liver, via the aforementioned neural gut-brain axis, which might take place in the situation of impaired renal glucose production, such as during CKD (Kaneko et al., 2018).

\section{Glucose phosphorylation and dephosphorylation.}

\section{$\underline{\text { Summary points }}$}

- This chapter focuses on glucose phosphorylation/dephosphorylation during the fed-tofasting state cycle in the liver and kidneys.

- Glucose-6 phosphate is the first intermediate of glucose metabolism.

- The postprandial elevation in G6P has a major role in stimulating glycogen storage.

- Glucokinase is the gatekeeper of glucose metabolism in hepatocytes and strongly regulates hepatic glycogen synthesis.

- Glucokinase activity is regulated by an inhibitory protein, GKRP, which sequesters glucokinase in the nucleus during fasting.

- In the kidneys, glucose phosphorylation is mediated by hexokinase. 
- Glucose-6 phosphatase is a key enzyme for endogenous glucose production. This enzyme ensures the last common step of both glycogenolysis and gluconeogenesis pathways i.e. the hydrolysis of glucose- 6 phosphate into glucose.

- Glucose-6 phosphatase is composed of two subunits (a G6P translocase, G6PT, and a catalytic subunit, G6PC), both localized in the membrane of the endoplasmic reticulum.

- The transcription factor ChREBP (carbohydrate-response element binding protein) is a central metabolic coordinator orchestrating the fate of glucose.

- Insulin-mediated lipogenesis is activated via the regulation of sterol regulatory elementbinding protein-1c (SREBP-1c), while glucose-mediated lipogenesis is activated via ChREBP.

In the liver and kidneys, extracellular and intracellular glucose concentrations are in equilibrium due in part to glucose transporter GLUT 2, characterized by a low affinity for glucose (15-20 mM) (Thorens, 1996) (Figure 1). Once in the cell, glucose is phosphorylated by glucokinase, specifically in the hepatocytes, or by hexokinases (Figure 1A). Glucokinase (GCK, also named hexokinase IV; encoded by Gck), exhibits kinetic properties quite distinct from those of other mammalian hexokinases (Agius, 2016). In vivo, the expression and/or activity of GCK is induced in response to an oral glucose and fructose load (Oosterveer et al., 2012; Van Schaftingen et al., 1994). GCK activity is regulated by an inhibitory protein, GKRP that sequesters GCK in the nucleus during fasting. The interaction of GCK and GKRP is also induced by fructose- 6 phosphate (Van Schaftingen et al., 1994). On the contrary, elevated blood glucose (above $5 \mathrm{mM}$ ) and fructose-1 phosphate enables dissociation of GCK and translocation in the cytoplasm, where GCK is active (Agius, 2016). It was recently shown that Sirtuin 2 (SIRT2) allows glucosedependent dissociation of GCK from GKRP by de-acetylating GKRP in the presence of elevated levels of NAD+ (Watanabe et al., 2018). Consistent with the major role of GCK in control of blood glucose homeostasis, transcription of Gck in the liver is induced by insulin through the 
transcription factor SREBP-1c and repressed by glucagon. Unlike other hexokinase, GCK is not inhibited by G6P and has relatively low affinity for glucose (Van Schaftingen et al., 1994). In addition, glucagon is predicted to favor sequestration of GCK in the nucleus and thereby increased hepatic glucose production and impaired hepatic glucose uptake (Agius, 2016). Thus, GCK enables hepatocytes to efficiently trap glucose when blood glucose is increased, playing a central role in glucose homeostasis. In the last decade, GCK activators and allosteric inhibitors of GCK-GKRP interaction have been proposed as a therapy for diabetes, with inconclusive results (Raimondo et al., 2015).

As mentioned above, G6P can enter three metabolic pathways including glycogen synthesis, glycolysis, and PPP as illustrated on Figure 1 (Adeva-Andany et al., 2016). Thus G6P may undergo isomerization into glucose-1 phosphate for glycogen synthesis, isomerization into fructose 6-phosphate and oxidation into gluconolactone, which initiates PPP. Fructose-6 phosphate may further lead to UDP-N-acetylglucosamine production (hexosamine pathway) or it can enter glycolysis to generate pyruvate and acetyl-CoA, via tricarboxylic acid cycle into the mitochondria. PPP is widely known to play a key role in reductive biosynthesis, given that this pathway is the major source of NADPH. While it was proposed that NADPH could be essential for glutathione reduction, it has been recently suggested that PPP is not an efficient pathway to protect the liver from oxidative stress (Jin et al., 2018). In addition, G6P and/or its metabolites (xylulose-5 phosphate or fructose-2,6-biphosphate) may act as signaling molecules to regulate transcription of specific glucose-responsive genes, by recruiting carbohydrate response element binding protein (ChREBP) and its partner Mlx (Abdul-Wahed et al., 2017; Adeva-Andany et al., 2016). ChREBP, which recognizes conserved carbohydrate response elements (ChoREs) in gene promoters, regulates genes involved in glycolysis ( $L p k$ ) and fatty acid synthesis (Fasn, Acaca, Scd1) (Benhamed et al., 2012; Dentin et al., 2012b). Recent data confirmed that the majority of ChREBP target genes are involved in metabolic processes in the liver and white adipose tissue, as well as in insulin signaling and tumorigenesis (Poungvarin et al., 2015). The various hormonal and nutritional regulations of ChREBP expression and activity during fasting and upon 
carbohydrate feeding have been detailed in a recent review (Abdul-Wahed et al., 2017). Thus, ChREBP is considered as a glucose sensor and a general integrator of metabolic signals according to nutritional states.

As soon as the intestinal absorption of glucose is completed (post-prandial period), hepatic G6P is mainly derived from glycogen breakdown, while GNG becomes the major source of G6P after prolonged fasting. In its phosphorylated form, glucose cannot be exported in the circulation and thus it is retained in the cell. In glucose producing organs, G6P is then dephosphorylated into glucose by G6Pase (Figure 1B). G6P is first translocated from the cytosol into the endoplasmic reticulum (ER) by the G6Pase transporter (G6PT encoded by SLC37A4), and subsequently dephosphorylated by the catalytic subunit of G6Pase (G6PC encoded by G6PC1). Both subunits are deeply embedded in the ER membrane and the catalytic site of G6PC faces the ER lumen. Glucose is finally released from the cytosol into the bloodstream through GLUT2. Indeed, GLUT2 deficiency leads to Fanconi-Bickel disease (GSD type XI) characterized by ketotic hypoglycemia during periods of fasting and hepatomegaly secondary to glycogen accumulation (Thorens, 2015). However, liver-GLUT2 deficiency in mice does not result in major perturbations in hepatic glucose production, which has suggested that glucose produced in the ER lumen could be released from a vesicle trafficking to the plasmatic membrane (Burcelin et al., 2000; Guillam et al., 1998). It is noteworthy that glucose release from the ER lumen by a pathway that would compartmentalize glucose away from the cytosol, where it could be rephosphorylated by GCK, could represent an interesting adaptation during fasting.

\section{Deregulation of glucose homeostasis in diabetes and glycogen storage disease type}

I.

Summary points 
- This chapter described two metabolic diseases, i.e. type 2 diabetes and glycogen storage disease type I, which are characterized by glucose metabolism impairments.

- Type 2 diabetes is characterized by an overproduction of glucose and hyperglycemia, while GSDI is characterized by a lack of endogenous glucose production and hypoglycemia.

- Type 2 diabetes and non-alcoholic fatty liver diseases (NAFLD) are chronic metabolic diseases that are associated with over-nutrition.

- GSDI is a rare genetic metabolic disease due to deficiency in G6Pase activity. GSD type Ia patients have mutations in G6PC, whereas GSD type Ib patients have mutations in G6P transporter (SLC37A4).

- When the storage capacity of glucose in the form of glycogen is exceeded, G6P is transformed into fat via the de novo lipogenesis pathway, leading to steatosis.

- Type 2 diabetes and GSDI patients both develop hepatic steatosis and long-term pathologies, including liver cancer and chronic kidney disease. In these diseases, hepatic and renal metabolism is characterized by an increased metabolism downstream of G6P.

- Lipid accumulation in the liver and kidneys play a key role in the occurrence of hepatic and renal damage.

- Hepatic steatosis is the major hallmark of NAFLD.

- The transcription factor ChREBP is a major mediator for promoting the conversion of dietary carbohydrates to fat for energy storage.

- In diabetes, NAFLD can progress to liver fibrosis and non-alcoholic steatohepatitis, and finally to the development of hepatocellular carcinomas (HCC). However, HCC can also occur from the transformation of hepatocellular adenomas (HCA) developed in fatty liver. In GSDI, most adult patients develop multiple HCA that can transform into HCC with time.

- In diabetes and GSDI, nephropathy can progress to interstitial fibrosis and glomerulosclerosis leading to renal failure. 
- A common metabolic feature of CKD and NAFLD is ectopic lipid accumulation, which is suspected to induce organ damage and dysfunction.

\subsection{Hepatic and renal metabolic disruption in type 2 diabetes and GSDI}

Type 2 diabetes is currently considered as an epidemic metabolic strongly linked to the development of obesity, which can be generated from a decrease in physical activity and an increase in caloric consumption in combination with genetic predisposition to insulin resistance (Chen et al., 2012). Indeed, type 2 diabetes is characterized by insulin resistance of the tissues capable of capturing glucose under the action of insulin and / or the lack of insulin production due to the decline in $\beta$-cell function. The lack of appropriate insulin signaling, associated with endogenous glucose overproduction leads to hyperglycemia (Lin and Accili, 2011). Thus the liver and kidney are chronically exposed to hyperglycemia. In the liver, it was shown that glucose uptake is impaired in obese diabetic mice, due to a decreased activity of SIRT2, blocking the dissociation of GCK and GKRP (Watanabe et al., 2018). This has also been associated with low hepatic GCK mRNA expression in diabetic patients (Haeusler et al., 2015). Indeed, high glucose represses the expression of the Gck gene and concomitantly induces G6pc gene expression (Arden et al., 2011). Thus glucose production is activated in diabetes, consequence an overall increase in GNG and of G6P production (Haeusler et al., 2015). This leads to aberrant glucose sensing that results in the accumulation of triglycerides (TG) in the liver and the development of steatosis via the concomitant activation of de novo lipogenesis and suppression of lipid oxidation. Thus diabetes, characterized by glucose metabolism dysfunction, is tightly linked to liver disease, more precisely to non-alcoholic fatty liver disease (NAFLD) (Bhatt and Smith, 2015; Hazlehurst et al., 2016). Indeed, up to 70\% of diabetic patients may present NAFLD (Hazlehurst et al., 2016; Williamson et al., 2011). Moreover, hypertriglyceridemia is a common lipid abnormality in persons with type 2 diabetes. In the kidneys, similar metabolic perturbations (increased de novo lipogenesis, decreased lipid oxidation, activation of 
hexosamine pathway...) are observed, leading to the development of chronic kidney disease (CKD) (Vallon and Komers, 2011), which will be described below.

GSDI (also known as the von Gierke's disease) is a rare genetic disease, with an incidence of 1/100,000 live births, due to a deficiency in G6Pase. Mutations in the gene encoding G6PC are responsible for GSD Type Ia and mutations in G6PT are responsible for GSD Type Ib (Bruni et al., 1999; Chevalier-Porst et al., 1996; Kishnani et al., 2014). Consequently, patients with GSDI cannot produce glucose during short fasts and may suffer from severe hypoglycemia episodes (Kishnani et al., 2014). The deficiency in G6Pase leads to the accumulation of G6P in the liver and kidneys, leading to a complete metabolic reprogramming, characterized in part by increased glycolysis and lipogenesis (Gjorgjieva et al., 2016a). Furthermore, we have recently demonstrated a strong reduction in hepatic GCK flux in GSDIa mice and an activation of glycogen turnover (Hijmans et al., 2017). GSDI patients show hepatomegaly and severe steatosis caused by strong glycogen and lipid accumulation, respectively. This is associated with lactic acidosis, hyperuricemia, hypercholesterolemia and hypertriglyceridemia(Bandsma et al., 2014; Kishnani et al., 2014). Furthermore, in these patients de novo lipogenesis and cholesterol synthesis were found to be increased 40-fold and 7-fold, respectively (Bandsma et al., 2008). Production of VLDL was unchanged compared to control values, but conversion of VLDL into intermediate density lipoproteins was relatively delayed in GSDI patients. Lipid vesicles are present in abundance in GSDI livers, mainly in the periportal zone, which corresponds to the location of the highest expression of G6Pase in the liver (Rajas et al., 2007). Associated with glycogen accumulation, lipids contribute further to the development of hepatomegaly. Recently, our results highlighted the crucial role that lipids also play in the development of CKD in GSDIa, independently of the occurrence of NAFLD (Monteillet et al., 2018). While hepatic manifestations of GSDI have been long known, CKD was acknowledged as a complication of GSDI later on, in the 1980's, when renal histology of GSDI patients revealed glomerulosclerosis, interstitial fibrosis and tubular atrophy (Kishnani et al., 2014). Indeed, G6Pase deficiency results in glycogen and lipid accumulation in renal proximal tubules of the kidney cortex. It has been 
reported that most adult GSDI patients over 25 years of age develop first signs of CKD, which can progress to renal failure with aging (Kishnani et al., 2014). Many patients also present nephrocalcinosis (due to hypercalciuria and hypocitraturia) that enhance the likelihood of urine calcium precipitation. Because of hyperuricemia, uric acid nephrolithiasis and gout nephropathy can develop. Unfortunately, the loss in renal function, glomerulosclerosis and interstitial fibrosis can further take place and these defects can lead to renal failure at later stage.

Thus, type 2 diabetes and GSDI share similar hepatic and renal metabolic dysfunctions leading to hepatic steatosis and CKD (Rajas et al., 2013).

\subsection{Deregulation of glucose homeostasis and hepatic steatosis}

When the storage capacity of glucose in the form of glycogen in the liver is exceeded, glucose is stored under the form of lipids. As previously mentioned, high G6P concentrations activate glycolysis, leading to the production of pyruvate. Pyruvate may then be transported in the mitochondria, where it is converted into acetyl-CoA, a precursor for the synthesis of fatty acids and cholesterol. Fatty acids can then be elongated, desaturated, esterified and used to synthetize TG. Triglycerides are stored in lipid vesicles in the cytosol, transported to the adipose tissue via the export of very low density lipoproteins (VLDL), or metabolized through the $\beta$-oxidation pathway (Postic and Girard, 2008). On a molecular level, lipid accumulation in the liver can be due to excessive lipid synthesis or import of fatty acids, a decrease in lipid degradation or problems in the export of VLDL (Postic and Girard, 2008). In insulin-resistant states, hyperglycemia and hyperinsulinemia are responsible for enhanced lipogenesis partly through the activation of ChREBP and SREBP-1c. Indeed, inhibition of ChREBP expression in obese diabetic ob/ob mice leads to reversal of hepatic steatosis (Dentin et al., 2006; Iizuka et al., 2006). In GSDI, lipid synthesis is activated by ChREBP, but independently of liver X receptor (LXR) and SREBP1c (Grefhorst et al., 2010). The lack of SREBP1c activation could be due to low insulin signaling in GSDI (Tong et al., 2009). Besides lipogenesis, ChREBP is known to potentiate glycolysis and nucleotide biosynthesis, and it can have an inhibitory role on oxidative 
phosphorylation (OXPHOS) (Tong et al., 2009). Thus ChREBP could be a metabolic switch orchestrating the metabolic reprogramming in GSDI and diabetic cells.

Contrarily to what is generally believed, there is no clear link between hepatic insulinresistance and fat storage in the liver. For example, despite severe hepatic steatosis, GSDIa mice are protected against diabetes induced by high-fat/high-sucrose diet (Abdul-Wahed et al., 2014). More likely, the consequences of hepatic steatosis are dependent on the specific nature of generated lipid species and/or their subcellular localization. The fractional contribution of hepatic de novo lipogenesis is also dependent on dietary carbohydrate intake. For example, fructose increases lipogenesis more strongly than glucose, through a significant activation of ChREBP (Kim et al., 2016). Moreover, this induces the expression of the hepatokine FGF21, which appears to exert a protective effect on the liver against steatosis (Fisher et al., 2017).

NAFLD encompasses a large spectrum of disease seriousness ranging from simple steatosis to inflammation, fibrosis, and potentially cirrhosis. Simple hepatic steatosis is thought to be a benign reversible condition characterized by the accumulation of $\mathrm{TG}$, in the absence of indicators of liver disease. However, NAFLD is a fertile ground for the development of hepatic tumors. In a subset of obese/diabetic individuals, NAFLD may transition to non-alcoholic steatohepatitis (NASH) characterized by the development of inflammation and fibrosis. However, hepatocarcinogenesis is also observed in non-fibrotic, non cirrhotic NAFLD livers (Baffy et al., 2012; Calzadilla Bertot and Adams, 2016). An uncertain fraction of tumors in nonfibrotic livers likely arise through transformation of HCA (Baffy et al., 2012; Stoot et al., 2010) (Figure 3). Interestingly, the same HCA-to-HCC transformation has been recently described in GSDI livers (Gjorgjieva et al., 2018). Despite the important levels of accumulated glycogen and lipids in the liver, as well as the important metabolic imbalance, GSDI patients present low-grade hepatic inflammation (Kishnani et al., 2014). Hepatic transaminase (AST / ALT) levels are also usually normal, especially in patients with optimal metabolic control and in patients not bearing hepatic tumors (Kim et al., 2008). The absence of oxidative stress and inflammatory responses 
in the case of GSDI might be the reason as to why hepatic fibrosis is not associated with GSDI (Kishnani et al., 2014). Indeed, GSDI patients and related animal models do not present fibrosis in the liver, contrarily to other types of glycogen storage diseases. Consequently, these patients do not develop cirrhosis. The comparison of HCC occurrence in NAFLD and GSDI livers argue for a dominant role of metabolic reprogramming in the molecular induction of tumor development. Recently, we showed that GSDIa hepatocytes exhibited the main characteristics of cancer cell metabolism, with a Warburg-like metabolic reprogramming that predisposes GSDIa livers to tumor development (Gjorgjieva et al., 2018). Indeed, we observed a hyperactivation of the glycolysis pathway characterized by an overexpression of the M2 isoform of pyruvate kinase in the tumors and an increase in lactate production. Moreover, OXPHOS analyses revealed a decrease in mitochondrial respiration with a reduction of pyruvate oxidation. This offers advantages to proliferating cells, providing substrates for cellular membrane biogenesis and amino acids and nucleotide synthesis for cell division. Altogether, these metabolic perturbations were associated with a decrease in cellular defenses, including decreased autophagy, chronic ER-stress and loss of tumor suppressors such as p53, HNF1 $\alpha$, PTEN (Gjorgjieva et al., 2016a, 2018). Interestingly, as observed in GSDIa, a decrease in autophagy levels has also been reported in other NAFLD (Koga et al., 2010; Mao et al., 2016; Yang et al., 2010). Furthermore, lipid accumulation and defective autophagy induce ER stress in NAFLD conditions (Yang et al., 2010). Interestingly, there is growing evidence indicating that ChREBP plays a central role in hepatocarcinogenesis. Indeed, the suppression of ChREBP in hepatoma and colorectal cancer cells led to a metabolic switch from aerobic glycolysis to mitochondrial respiration coupled with reduced lipogenesis and nucleotide synthesis and decreased proliferative and tumorigenic potential in vivo (Tong et al., 2009). It was also shown that advanced glycation end products (AGEs) that are elevated in diabetic patients stimulate both the expression and activity of ChREBP and promote cancer cell proliferation (Chen et al., 2014). In GSDIa, the overexpression of ChREBP has been linked to glucose and lipid metabolism reprogramming (Abdul-Wahed et al., 2014; Gjorgjieva et al., 2018). In this context, enhanced ChREBP could partly account for 
increased proliferation of hepatocytes by favoring cancer cell metabolism. Further investigation is required to unravel the exact role of ChREBP in hepatocarcinogenesis in the context of NAFLD.

In conclusion, both diabetic and GSDI patients develop severe hepatic steatosis and are prone to the development of hepatic tumors. It is noteworthy that tumor occurrence is higher in GSDI patients than in diabetes. Interestingly, molecular mechanisms involved in hepatocarcinogenesis in this steatotic context seem different from HCC induced by some viral infections and alcoholic liver diseases. By comparing hepatocarcinogenesis in diabetes and GSDI, we can propose a dominant role of hepatic metabolic reprogramming in this process. Thus, a rational therapeutic approach for the treatment of NAFLD, before the development of hepatic tumors, could be to increase hepatic energy expenditure and thereby increase hepatic fat oxidation. Recently, it was shown that T3-glucagon conjugate or glucacon like peptide 1 agonistgastric inhibitory peptide-glucagon tri-conjugate improved glycemia and NAFLD in diabetes, without the harmful effects of each hormone (Finan et al., 2015, 2016). Liver-targeted mitochondrial uncouplers, which can dissipate the inner mitochondrial membrane proton gradient in the liver are also a promising class of agent for the treatment of NAFLD in the context of diabetes (Perry et al., 2015). Interestingly, lipid-lowering treatments that increased fatty acid oxidation by using pharmacological activators of PPAR $\alpha$ have recently shown to prevent hepatic damages in both diabetes and GSDI (Monteillet et al., 2018; Musso et al., 2016; Waskowicz et al., 2018) .

\subsection{Deregulation of glucose homeostasis and chronic kidney disease}

The molecular mechanism leading to CKD in GSDI patients is thought to be very similar to the one observed in diabetic patients (Mundy and Lee, 2002; Rajas et al., 2013; Yiu et al., 2008). Indeed, the increased flux downstream of G6P leads to the accumulation of ectopic lipids in the kidneys (Figure 4). This results in the activation of the Renin-Angiotensin system (RAS) 
observed in both diabetic and GSDI CKD (Mundy and Lee, 2002; Yiu et al., 2008). Lipids are precursors for the de novo synthesis of glycerolipids, including diacylglycerol (DAG). DAG is a potent activator of protein kinase C (PKC) and acts through binding to a specific domain of the regulatory subunit of the enzyme. The activation of PKC leads to the activation of RAS. In RAS, angiotensinogen (Agt) is the precursor of Angiotensin II (Ang II) that is produced from two sequential enzymatic reactions: the conversion of Agt in Angiotensin I by renin and the conversion of Ang I in Ang II by Ang converting enzyme (ACE). Ang II stimulates the proliferation of mesangial cells, glomerular endothelial cells, and fibroblasts, and acts as a profibrogenic factor. Many profibrotic effects of RAS are mediated by transforming growth factor- $\beta 1$ (TGF- $\beta 1$ ). Indeed, renal fibrosis development is induced by TGF- $\beta 1$, which activates the expression of the extracellular matrix genes (Lan, 2011; Meng et al., 2015). In addition, TGF$\beta 1$ is a potent inducer of epithelial-to-mesenchymal transition that characterizes the phenotype of renal interstitial fibrosis. Thus several treatments inhibiting the RAS / TGF- $\beta 1$ axis, such as ACE inhibitors, are used to retard progression of CKD in diabetic and GSDI patients (Gjorgjieva et al., 2016b; Martens et al., 2009; Patney et al., 2018). Interestingly, as lipids are a major contributor to the development of CKD, lipid-lowering drugs, such as fenofibrate via the activation of PPAR $\alpha$, could be suitable therapeutic strategy to prevent nephropathy. Some studies already evoked renoprotective effects of fenofibrate in diabetes and GSDI (Lewis and Wanner, 2012; Monteillet et al., 2018; Ting et al., 2012). Unfortunately, while lipid-lowering effects of fibrate were corrective of both pathologies featuring the liver and kidneys of GSDIa mice, this strategy did not insure normal glycemia (Monteillet et al., 2018).

To conclude, by comparing hepatic and renal diseases in diabetes and GSDI, we emphasize the striking similarities of molecular metabolism dysfunction in these two diseases. A stimulated metabolism down-stream of G6P is a common causal feature leading to the accumulation of ectopic lipids in the liver and kidneys. In the liver, hepatic steatosis results in HCA/HCC development with time. In the kidneys, renal lipids are responsible for kidney injuries, finally responsible for the loss of renal filtration function. It is a noteworthy observation that, 
while diabetes and GSDI are characterized by abnormal glucose metabolism, lipid-lowering drugs might represent a potential therapeutic approach to prevent both NAFLD and CKD development in both diabetes and GSDI.

\section{Acknowledgements}

The authors thank all past and current colleagues in the laboratory who contributed to the work reviewed.

\section{Legends of figures}

Figure 1: Schematic glucose metabolism in hepatocytes in the fed state and postabsorptive state. Under fed conditions (A), glucose promotes the dissociation of GCK from GKRP, which leads to the cytoplasmic translocation of GCK. Thus, glucose is phosphorylated into G6P, which can enter three metabolic pathways including glycogen synthesis, glycolysis, and PPP. Glucose is then stored under the form of glycogen. G6P is also degraded during glycolysis, providing energy and pyruvate in further decarboxylated to acetyl-CoA, which enters the intramitochondrial tricarboxyl acid cycle (TCA). Alternatively, G6P is degraded in PPP, which provides NADPH and nucleotides. When glucose is in excess, de novo lipogenesis is activated and G6P is transformed into fat leading to steatosis. (B) Under fasted conditions, glycogenolysis and gluconeogenesis provide G6P as a substrate for glucose production. G6P is then dephosphorylated by G6Pase, which is localized in endoplasmic reticulum. In the liver, glycerol, lactate and alanine are used as gluconeogenic substrates. Moreover, GCK is sequestered in the nucleus, bound to GKRP, limiting the phosphorylation of glucose. GLUT2: glucose transporter 2; GCK: glucokinase; G6Pase, glucose-6 phosphatase; G6P: glucose-6 phosphate; F6P, fructose-6 
phosphate, PPP: pentose phosphate pathway. Illustrations were done with the help of Servier Medical Art.

Figure 2: Development of chronic kidney disease and hepatic tumors in type 2 diabetes and GSDI. Type 2 diabetes and GSDI are two metabolic diseases characterized by glucose metabolism impairments. While type 2 diabetes is characterized by an overproduction of glucose and hyperglycemia, GSDI is due to an absence of glucose production leading to hypoglycemia. Thus, these two pathologies could be considered as "mirror" diseases. However, lipids are accumulated in the liver and kidneys of type 2 diabetes and GSDI patients, which is responsible for the development of NAFLD and CKD. With time, renal failure and hepatic tumors can occur. CKD: chronic kidney disease; NAFLD: non-alcoholic fatty liver disease. Illustrations were done with the help of Servier Medical Art.

Figure 3: Schematic steps of the development of hepatocellular adenomas and carcinomas. Hepatic steatosis is the major hallmark of NAFLD-like pathologies, which can lead to the development of hepatic tumors. In most cases, NAFLD progresses to non-alcoholic steatohepatitis (NASH) due to inflammation and fibrosis, and ultimately to HCC. However, the development of HCC is more and more observed after the occurrence of HCA in the absence of inflammation and fibrosis. HCA: hepatocellular adenomas; HCC: hepatocellular carcinomas. Illustrations were done with the help of Servier Medical Art.

Figure 4: CKD progression until the end-stage kidney disease. After the first alterations of kidneys, hyperfiltration is often observed before the progressive lack of renal function (decreased glomerular filtration rate). In type 2 diabetes and GSDI, the accumulation of lipids in the renal cortex induces interstitial fibrosis and glomerulosclerosis, responsible of CKD. Then, 
the progression of CKD into renal failure requires dialysis and then kidney transplantation. Illustrations were done with the help of Servier Medical Art.

\section{References}

Abdul-Wahed, A., Gautier-Stein, A., Casteras, S., Soty, M., Roussel, D., Romestaing, C., Guillou, H., Tourette, J.-A., Pleche, N., Zitoun, C., Gri, B., Sardella, A., Rajas, F., Mithieux, G., 2014. A link between hepatic glucose production and peripheral energy metabolism via hepatokines. Mol Metab 3, 531-543.

Abdul-Wahed, A., Guilmeau, S., Postic, C., 2017. Sweet Sixteenth for ChREBP: Established Roles and Future Goals. Cell Metab. 26, 324-341.

Adeva-Andany, M.M., Pérez-Felpete, N., Fernández-Fernández, C., Donapetry-García, C., PazosGarcía, C., 2016. Liver glucose metabolism in humans. Biosci. Rep. 36.

Agius, L., 2016. Hormonal and Metabolite Regulation of Hepatic Glucokinase. Annu. Rev. Nutr. 36, 389-415.

Agius, L., 2015. Role of glycogen phosphorylase in liver glycogen metabolism. Molecular Aspects of Medicine 46, 34-45.

Arden, C., Petrie, J.L., Tudhope, S.J., Al-Oanzi, Z., Claydon, A.J., Beynon, R.J., Towle, H.C., Agius, L., 2011. Elevated Glucose Represses Liver Glucokinase and Induces Its Regulatory Protein to Safeguard Hepatic Phosphate Homeostasis. Diabetes 60, 3110-3120.

Baffy, G., Brunt, E.M., Caldwell, S.H., 2012. Hepatocellular carcinoma in non-alcoholic fatty liver disease: An emerging menace. Journal of Hepatology 56, 1384-1391.

Bandsma, R.H.J., Prinsen, B.H., de Sain-van der Velden, M., Rake, J.-P., Boer, T., Smit, G.P.A., Reijngoud, D.-J., Kuipers, F., 2008. Increased de novo Lipogenesis and Delayed Conversion of 
Large VLDL into Intermediate Density Lipoprotein Particles Contribute to Hyperlipidemia in Glycogen Storage Disease Type 1a. Pediatr Res 63, 702-707.

Bandsma, R.H.J., Smit, G.P.A., Kuipers, F., 2014. Disturbed lipid metabolism in glycogen storage disease type 1. Eur J Pediatr 161, S65-S69.

Benhamed, F., Denechaud, P.-D., Lemoine, M., Robichon, C., Moldes, M., Bertrand-Michel, J., Ratziu, V., Serfaty, L., Housset, C., Capeau, J., Girard, J., Guillou, H., Postic, C., 2012. The lipogenic transcription factor ChREBP dissociates hepatic steatosis from insulin resistance in mice and humans. J. Clin. Invest. 122, 2176-2194.

Berry, G.T., Baker, L., Kaplan, F.S., Witzleben, C.L., 1995. Diabetes-like renal glomerular disease in Fanconi-Bickel syndrome. Pediatr. Nephrol. 9, 287-291.

Bhatt, H.B., Smith, R.J., 2015. Fatty liver disease in diabetes mellitus. Hepatobiliary Surg Nutr 4, 101-108.

Bruni, N., Rajas, F., Montano, S., Chevalier-Porst, F., Maire, I., Mithieux, G., 1999. Enzymatic characterization of four new mutations in the glucose-6 phosphatase (G6PC) gene which cause glycogen storage disease type 1a. Ann. Hum. Genet. 63, 141-146.

Burcelin, R., del Carmen Muñoz, M., Guillam, M.T., Thorens, B., 2000. Liver hyperplasia and paradoxical regulation of glycogen metabolism and glucose-sensitive gene expression in GLUT2null hepatocytes. Further evidence for the existence of a membrane-based glucose release pathway. J. Biol. Chem. 275, 10930-10936.

Calzadilla Bertot, L., Adams, L.A., 2016. The Natural Course of Non-Alcoholic Fatty Liver Disease. Int J Mol Sci 17.

Chen, H., Wu, L., Li, Y., Meng, J., Lin, N., Yang, D., Zhu, Y., Li, X., Li, M., Xu, Y., Wu, Y., Tong, X., Su, Q., 2014. Advanced glycation end products increase carbohydrate responsive element binding protein expression and promote cancer cell proliferation. Mol. Cell. Endocrinol. 395, 69-78. 
Chen, L., Magliano, D.J., Zimmet, P.Z., 2012. The worldwide epidemiology of type 2 diabetes mellitus--present and future perspectives. Nat Rev Endocrinol 8, 228-236.

Chevalier-Porst, F., Bozon, D., Bonardot, A.M., Bruni, N., Mithieux, G., Mathieu, M., Maire, I., 1996. Mutation analysis in 24 French patients with glycogen storage disease type 1a. J. Med. Genet. 33, $358-360$.

Croset, M., Rajas, F., Zitoun, C., Hurot, J.M., Montano, S., Mithieux, G., 2001. Rat small intestine is an insulin-sensitive gluconeogenic organ. Diabetes 50, 740-746.

Dentin, R., Benhamed, F., Hainault, I., Fauveau, V., Foufelle, F., Dyck, J.R.B., Girard, J., Postic, C., 2006. Liver-specific inhibition of ChREBP improves hepatic steatosis and insulin resistance in ob/ob mice. Diabetes 55, 2159-2170.

Dentin, R., Tomas-Cobos, L., Foufelle, F., Leopold, J., Girard, J., Postic, C., Ferré, P., 2012. Glucose 6phosphate, rather than xylulose 5-phosphate, is required for the activation of ChREBP in response to glucose in the liver. J. Hepatol. 56, 199-209.

Donovan, C.M., Watts, A.G., 2014. Peripheral and central glucose sensing in hypoglycemic detection. Physiology (Bethesda) 29, 314-324.

Finan, B., Clemmensen, C., Zhu, Z., Stemmer, K., Gauthier, K., Müller, L., De Angelis, M., Moreth, K., Neff, F., Perez-Tilve, D., Fischer, K., Lutter, D., Sánchez-Garrido, M.A., Liu, P., Tuckermann, J., Malehmir, M., Healy, M.E., Weber, A., Heikenwalder, M., Jastroch, M., Kleinert, M., Jall, S., Brandt, S., Flamant, F., Schramm, K.-W., Biebermann, H., Döring, Y., Weber, C., Habegger, K.M., Keuper, M., Gelfanov, V., Liu, F., Köhrle, J., Rozman, J., Fuchs, H., Gailus-Durner, V., Hrabě de Angelis, M., Hofmann, S.M., Yang, B., Tschöp, M.H., DiMarchi, R., Müller, T.D., 2016. Chemical Hybridization of Glucagon and Thyroid Hormone Optimizes Therapeutic Impact for Metabolic Disease. Cell 167, 843-857.e14.

Finan, B., Yang, B., Ottaway, N., Smiley, D.L., Ma, T., Clemmensen, C., Chabenne, J., Zhang, L., 
Habegger, K.M., Fischer, K., Campbell, J.E., Sandoval, D., Seeley, R.J., Bleicher, K., Uhles, S., Riboulet, W., Funk, J., Hertel, C., Belli, S., Sebokova, E., Conde-Knape, K., Konkar, A., Drucker, D.J., Gelfanov, V., Pfluger, P.T., Müller, T.D., Perez-Tilve, D., DiMarchi, R.D., Tschöp, M.H., 2015. A rationally designed monomeric peptide triagonist corrects obesity and diabetes in rodents. Nat. Med. 21, 27-36.

Fisher, F.M., Kim, M., Doridot, L., Cunniff, J.C., Parker, T.S., Levine, D.M., Hellerstein, M.K., Hudgins, L.C., Maratos-Flier, E., Herman, M.A., 2017. A critical role for ChREBP-mediated FGF21 secretion in hepatic fructose metabolism. Mol Metab 6, 14-21.

Gerich, J.E., Meyer, C., Woerle, H.J., Stumvoll, M., 2001. Renal gluconeogenesis: its importance in human glucose homeostasis. Diabetes Care 24, 382-391.

Gjorgjieva, M., Calderaro, J., Monteillet, L., Silva, M., Raffin, M., Brevet, M., Romestaing, C., Roussel, D., Zucman-Rossi, J., Mithieux, G., Rajas, F., 2018. Dietary exacerbation of metabolic stress leads to accelerated hepatic carcinogenesis in glycogen storage disease type Ia. J. Hepatol. https://doi.org/10.1016/j.jhep.2018.07.017

Gjorgjieva, M., Oosterveer, M.H., Mithieux, G., Rajas, F., 2016a. Mechanisms by Which Metabolic Reprogramming in GSD1 Liver Generates a Favorable Tumorigenic Environment. Journal of Inborn Errors of Metabolism and Screening 4, https://doi.org/10.1177/2326409816679429

Gjorgjieva, M., Raffin, M., Duchampt, A., Perry, A., Stefanutti, A., Brevet, M., Tortereau, A., Dubourg, L., Hubert-Buron, A., Mabille, M., Pelissou, C., Lassalle, L., Labrune, P., Mithieux, G., Rajas, F., 2016b. Progressive development of renal cysts in glycogen storage disease type I. Hum. Mol. Genet. 25, 3784-3797.

Grefhorst, A., Schreurs, M., Oosterveer, M.H., Cortés, V.A., Havinga, R., Herling, A.W., Reijngoud, D.-J., Groen, A.K., Kuipers, F., 2010. Carbohydrate-response-element-binding protein (ChREBP) and not the liver X receptor $\alpha(\operatorname{LXR} \alpha)$ mediates elevated hepatic lipogenic gene expression in a mouse model of glycogen storage disease type 1 . Biochem. J. 432, 249-254. 
Guillam, M.T., Burcelin, R., Thorens, B., 1998. Normal hepatic glucose production in the absence of GLUT2 reveals an alternative pathway for glucose release from hepatocytes. Proc. Natl. Acad. Sci. U.S.A. 95, 12317-12321.

Haeusler, R.A., Camastra, S., Astiarraga, B., Nannipieri, M., Anselmino, M., Ferrannini, E., 2015. Decreased expression of hepatic glucokinase in type 2 diabetes. Mol Metab 4, 222-226.

Hazlehurst, J.M., Woods, C., Marjot, T., Cobbold, J.F., Tomlinson, J.W., 2016. Non-alcoholic fatty liver disease and diabetes. Metabolism 65, 1096-1108.

Hicks, J., Wartchow, E., Mierau, G., 2011. Glycogen storage diseases: a brief review and update on clinical features, genetic abnormalities, pathologic features, and treatment. Ultrastruct Pathol 35, 183-196.

Hijmans, B.S., Boss, A., van Dijk, T.H., Soty, M., Wolters, H., Mutel, E., Groen, A.K., Derks, T.G.J., Mithieux, G., Heerschap, A., Reijngoud, D.-J., Rajas, F., Oosterveer, M.H., 2017. Hepatocytes contribute to residual glucose production in a mouse model for glycogen storage disease type Ia. Hepatology 66, 2042-2054.

Iizuka, K., Miller, B., Uyeda, K., 2006. Deficiency of carbohydrate-activated transcription factor ChREBP prevents obesity and improves plasma glucose control in leptin-deficient (ob/ob) mice. Am. J. Physiol. Endocrinol. Metab. 291, E358-364.

Jiang, G., Zhang, B.B., 2003. Glucagon and regulation of glucose metabolism. American Journal of Physiology - Endocrinology and Metabolism 284, E671-E678.

Jin, E.S., Lee, M.H., Murphy, R.E., Malloy, C.R., 2018. Pentose phosphate pathway activity parallels lipogenesis but not antioxidant processes in rat liver. American Journal of PhysiologyEndocrinology and Metabolism 314, E543-E551.

Kaneko, K., Soty, M., Zitoun, C., Duchampt, A., Silva, M., Philippe, E., Gautier-Stein, A., Rajas, F., Mithieux, G., 2018. The role of kidney in the inter-organ coordination of endogenous glucose 
production during fasting. Mol Metab.

Kim, M.-S., Krawczyk, S.A., Doridot, L., Fowler, A.J., Wang, J.X., Trauger, S.A., Noh, H.-L., Kang, H.J., Meissen, J.K., Blatnik, M., Kim, J.K., Lai, M., Herman, M.A., 2016. ChREBP regulates fructoseinduced glucose production independently of insulin signaling. Journal of Clinical Investigation $126,4372-4386$.

Kim, S.Y., Weinstein, D.A., Starost, M.F., Mansfield, B.C., Chou, J.Y., 2008. Necrotic foci, elevated chemokines and infiltrating neutrophils in the liver of glycogen storage disease type Ia. J. Hepatol. 48, 479-485.

Kishnani, P.S., Austin, S.L., Abdenur, J.E., Arn, P., Bali, D.S., Boney, A., Chung, W.K., Dagli, A.I., Dale, D., Koeberl, D., Somers, M.J., Wechsler, S.B., Weinstein, D.A., Wolfsdorf, J.I., Watson, M.S., American College of Medical Genetics and Genomics, 2014. Diagnosis and management of glycogen storage disease type I: a practice guideline of the American College of Medical Genetics and Genomics. Genet. Med. 16, e1.

Koga, H., Kaushik, S., Cuervo, A.M., 2010. Altered lipid content inhibits autophagic vesicular fusion. FASEB J 24, 3052-3065.

Lan, H.Y., 2011. Diverse Roles of TGF- $\beta$ /Smads in Renal Fibrosis and Inflammation. Int J Biol Sci 7, 1056-1067.

Lewis, D., Wanner, C., 2012. Diabetes: Should we use fibrates in patients with diabetes and mild CKD? Nature Reviews Nephrology 8, 201-202.

Lin, H.V., Accili, D., 2011. Hormonal regulation of hepatic glucose production in health and disease. Cell Metab. 14, 9-19.

Mao, Y., Yu, F., Wang, J., Guo, C., Fan, X., 2016. Autophagy: a new target for nonalcoholic fatty liver disease therapy. Hepat Med 8, 27-37.

Martens, D.H.J., Rake, J.P., Navis, G., Fidler, V., van Dael, C.M.L., Smit, G.P.A., 2009. Renal Function 
in Glycogen Storage Disease Type I, Natural Course, and Renopreservative Effects of ACE Inhibition. Clin J Am Soc Nephrol 4, 1741-1746.

Meng, X.-M., Tang, P.M.-K., Li, J., Lan, H.Y., 2015. TGF- $\beta /$ Smad signaling in renal fibrosis. Front Physiol 6.

Monteillet, L., Gjorgjieva, M., Silva, M., Verzieux, V., Imikirene, L., Duchampt, A., Guillou, H., Mithieux, G., Rajas, F., 2018. Intracellular lipids are an independent cause of liver injury and chronic kidney disease in non alcoholic fatty liver disease-like context. Mol Metab. https://doi.org/10.1016/j.molmet.2018.07.006

Mundy, H.R., Lee, P.J., 2002. Glycogenosis type I and diabetes mellitus: a common mechanism for renal dysfunction? Med. Hypotheses 59, 110-114.

Musso, G., Cassader, M., Gambino, R., 2016. Non-alcoholic steatohepatitis: emerging molecular targets and therapeutic strategies. Nat Rev Drug Discov 15, 249-274.

Mutel, E., Gautier-Stein, A., Abdul-Wahed, A., Amigó-Correig, M., Zitoun, C., Stefanutti, A., Houberdon, I., Tourette, J.-A., Mithieux, G., Rajas, F., 2011. Control of Blood Glucose in the Absence of Hepatic Glucose Production During Prolonged Fasting in Mice. Diabetes 60, 31213131.

Oosterveer, M.H., Mataki, C., Yamamoto, H., Harach, T., Moullan, N., van Dijk, T.H., Ayuso, E., Bosch, F., Postic, C., Groen, A.K., Auwerx, J., Schoonjans, K., 2012. LRH-1-dependent glucose sensing determines intermediary metabolism in liver. J. Clin. Invest. 122, 2817-2826.

Owen, O.E., Felig, P., Morgan, A.P., Wahren, J., Cahill, G.F., 1969. Liver and kidney metabolism during prolonged starvation. J. Clin. Invest. 48, 574-583.

Patney, V., Chaudhary, K., Whaley-Connell, A., 2018. Treatment of Diabetic Kidney Disease With Hypertension Control and Renin Angiotensin System Inhibition. Adv Chronic Kidney Dis 25, 158-165. https://doi.org/10.1053/j.ackd.2017.11.002 
Penhoat, A., Fayard, L., Stefanutti, A., Mithieux, G., Rajas, F., 2014. Intestinal gluconeogenesis is crucial to maintain a physiological fasting glycemia in the absence of hepatic glucose production in mice. Metab. Clin. Exp. 63, 104-111.

Perry, R.J., Zhang, D., Zhang, X.-M., Boyer, J.L., Shulman, G.I., 2015. Controlled-release mitochondrial protonophore reverses diabetes and steatohepatitis in rats. Science 347, 12531256.

Pillot, B., Soty, M., Gautier-Stein, A., Zitoun, C., Mithieux, G., 2009. Protein feeding promotes redistribution of endogenous glucose production to the kidney and potentiates its suppression by insulin. Endocrinology 150, 616-624.

Postic, C., Girard, J., 2008. Contribution of de novo fatty acid synthesis to hepatic steatosis and insulin resistance: lessons from genetically engineered mice. J. Clin. Invest. 118, 829-838.

Poungvarin, N., Chang, B., Imamura, M., Chen, J., Moolsuwan, K., Sae-Lee, C., Li, W., Chan, L., 2015. Genome-Wide Analysis of ChREBP Binding Sites on Male Mouse Liver and White Adipose Chromatin. Endocrinology 156, 1982-1994.

Raimondo, A., Rees, M.G., Gloyn, A.L., 2015. Glucokinase regulatory protein: complexity at the crossroads of triglyceride and glucose metabolism. Curr. Opin. Lipidol. 26, 88-95.

Rajas, F., Jourdan-Pineau, H., Stefanutti, A., Mrad, E.A., Iynedjian, P.B., Mithieux, G., 2007. Immunocytochemical localization of glucose 6-phosphatase and cytosolic phosphoenolpyruvate carboxykinase in gluconeogenic tissues reveals unsuspected metabolic zonation. Histochem. Cell Biol. 127, 555-565.

Rajas, F., Labrune, P., Mithieux, G., 2013. Glycogen storage disease type 1 and diabetes: learning by comparing and contrasting the two disorders. Diabetes Metab. 39, 377-387.

Roach, P.J., Depaoli-Roach, A.A., Hurley, T.D., Tagliabracci, V.S., 2012. Glycogen and its metabolism: some new developments and old themes. Biochem. J. 441, 763-787. 
Soty, M., Gautier-Stein, A., Rajas, F., Mithieux, G., 2017. Gut-Brain Glucose Signaling in Energy Homeostasis. Cell Metabolism 25, 1231-1242.

Stoot, J.H.M.B., Coelen, R.J.S., De Jong, M.C., Dejong, C.H.C., 2010. Malignant transformation of hepatocellular adenomas into hepatocellular carcinomas: a systematic review including more than 1600 adenoma cases. HPB (Oxford) 12, 509-522.

Thorens, B., 2015. GLUT2, glucose sensing and glucose homeostasis. Diabetologia 58, 221-232.

Thorens, B., 1996. Glucose transporters in the regulation of intestinal, renal, and liver glucose fluxes. Am. J. Physiol. 270, G541-553.

Ting, R.-D., Keech, A.C., Drury, P.L., Donoghoe, M.W., Hedley, J., Jenkins, A.J., Davis, T.M.E., Lehto, S., Celermajer, D., Simes, R.J., Rajamani, K., Stanton, K., FIELD Study Investigators, 2012. Benefits and safety of long-term fenofibrate therapy in people with type 2 diabetes and renal impairment: the FIELD Study. Diabetes Care 35, 218-225.

Tong, X., Zhao, F., Mancuso, A., Gruber, J.J., Thompson, C.B., 2009. The glucose-responsive transcription factor ChREBP contributes to glucose-dependent anabolic synthesis and cell proliferation. Proc. Natl. Acad. Sci. U.S.A. 106, 21660-21665.

Vallon, V., Komers, R., 2011. Pathophysiology of the diabetic kidney. Compr Physiol 1, 11751232.

Van Schaftingen, E., Detheux, M., Veiga da Cunha, M., 1994. Short-term control of glucokinase activity: role of a regulatory protein. FASEB J. 8, 414-419.

Waskowicz, L.R., Zhou, J., Landau, D.J., Brooks, E.D., Lim, A., Yavarow, Z.A., Kudo, T., Zhang, H., Wu, Y., Grant, S., Young, S.P., Huat, B.B., Yen, P.M., Koeberl, D.D., 2018. Bezafibrate induces autophagy and improves hepatic lipid metabolism in glycogen storage disease type Ia. Hum. Mol. Genet. https://doi.org/10.1093/hmg/ddy343

Watanabe, H., Inaba, Y., Kimura, K., Matsumoto, M., Kaneko, S., Kasuga, M., Inoue, H., 2018. Sirt2 
facilitates hepatic glucose uptake by deacetylating glucokinase regulatory protein. Nat Commun 9,30 .

Weinstein, D.A., Steuerwald, U., De Souza, C.F.M., Derks, T.G.J., 2018. Inborn Errors of Metabolism with Hypoglycemia: Glycogen Storage Diseases and Inherited Disorders of Gluconeogenesis. Pediatr. Clin. North Am. 65, 247-265.

Williamson, R.M., Price, J.F., Glancy, S., Perry, E., Nee, L.D., Hayes, P.C., Frier, B.M., Van Look, L.A.F., Johnston, G.I., Reynolds, R.M., Strachan, M.W.J., Edinburgh Type 2 Diabetes Study Investigators, 2011. Prevalence of and risk factors for hepatic steatosis and nonalcoholic Fatty liver disease in people with type 2 diabetes: the Edinburgh Type 2 Diabetes Study. Diabetes Care 34, 11391144.

Yang, L., Li, P., Fu, S., Calay, E.S., Hotamisligil, G.S., 2010. Defective Hepatic Autophagy in Obesity Promotes ER Stress and Causes Insulin Resistance. Cell Metab 11, 467-478.

Yiu, W.H., Pan, C.-J., Ruef, R.A., Peng, W.-T., Starost, M.F., Mansfield, B.C., Chou, J.Y., 2008. The angiotensin system mediates renal fibrosis in glycogen storage disease type Ia nephropathy. Kidney Int 73, 716-723. 
A- Fed state

Glucose
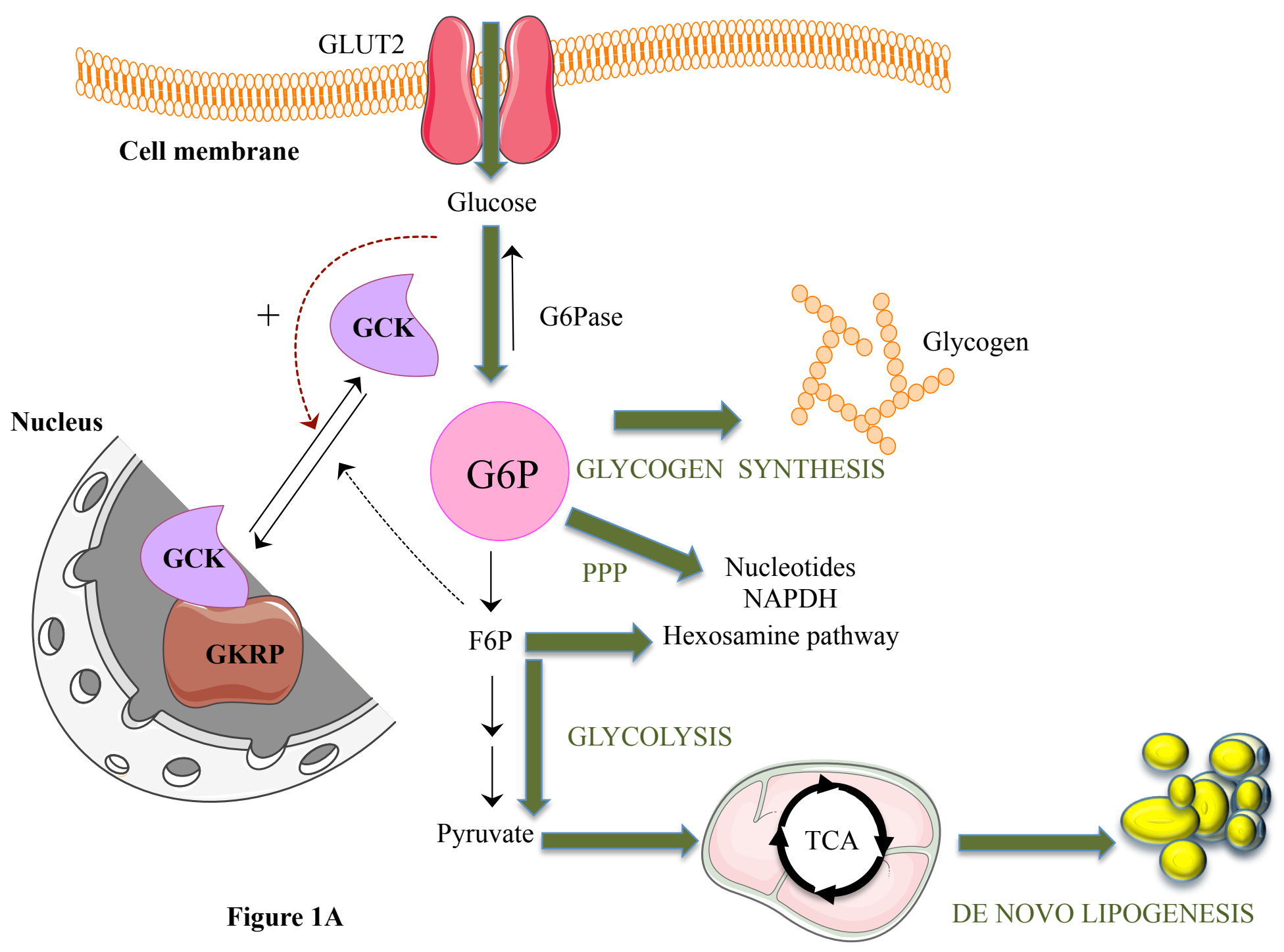
B- Fasted state Glucose
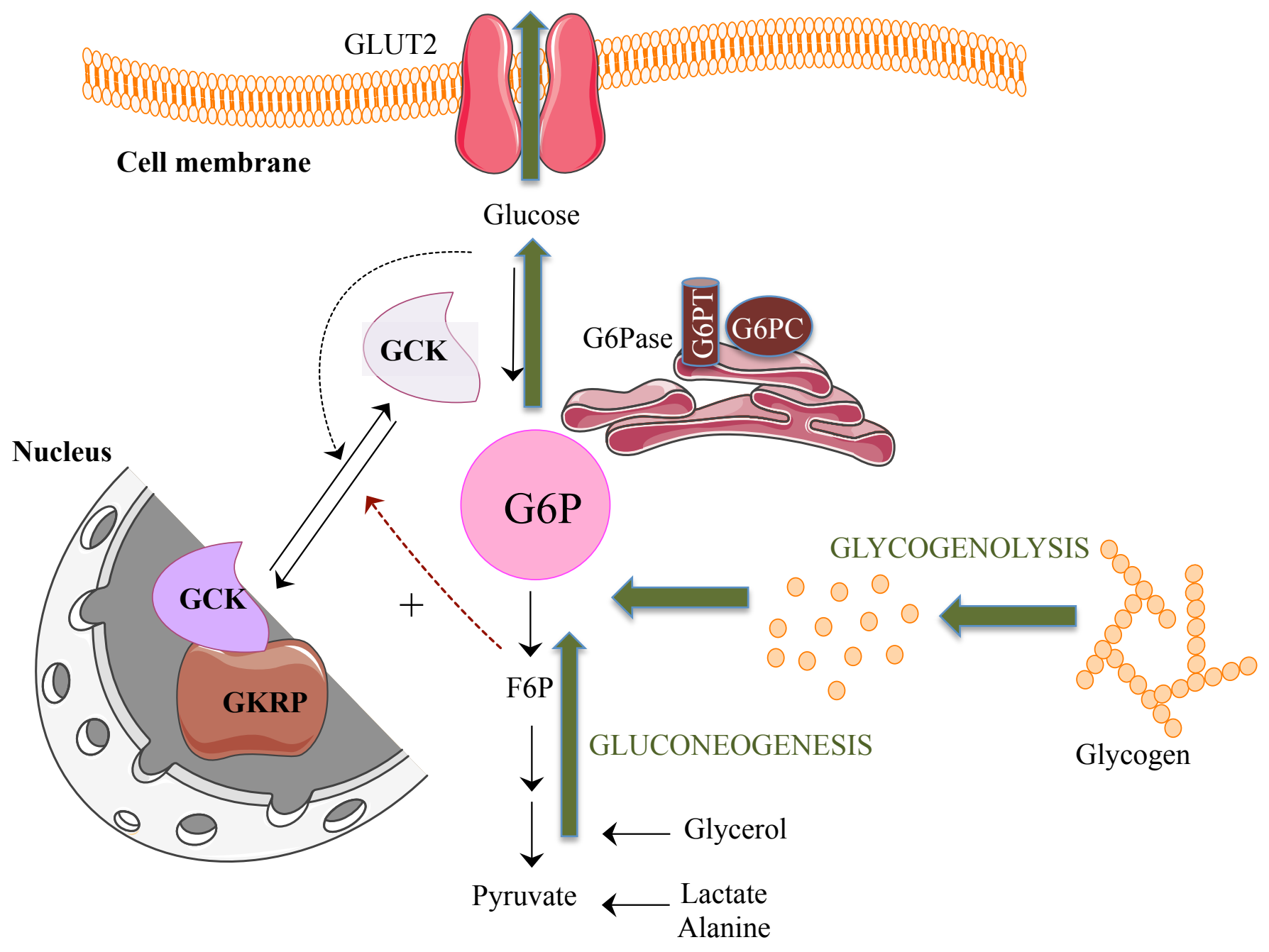

Figure 1B 


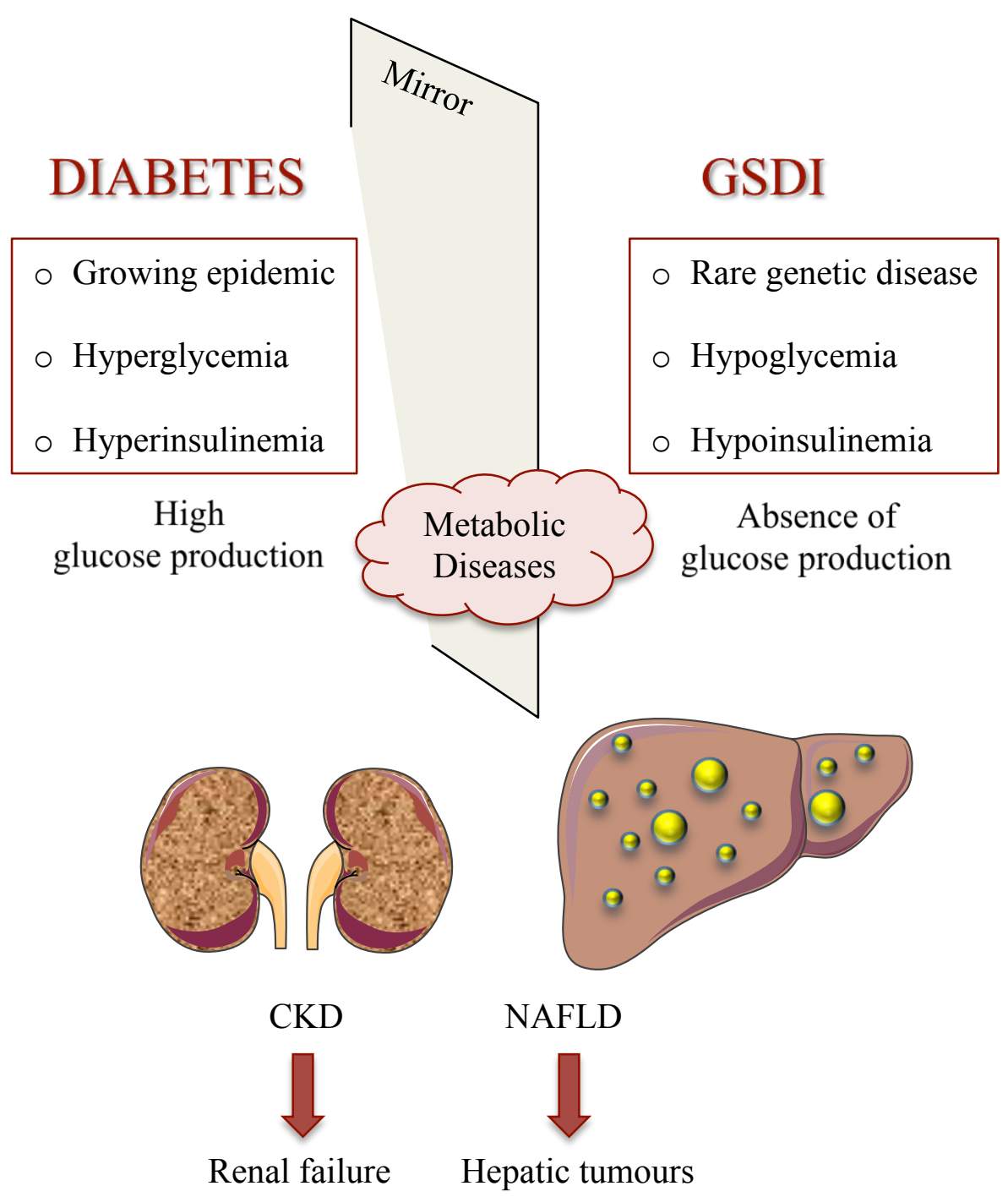

Figure 2 

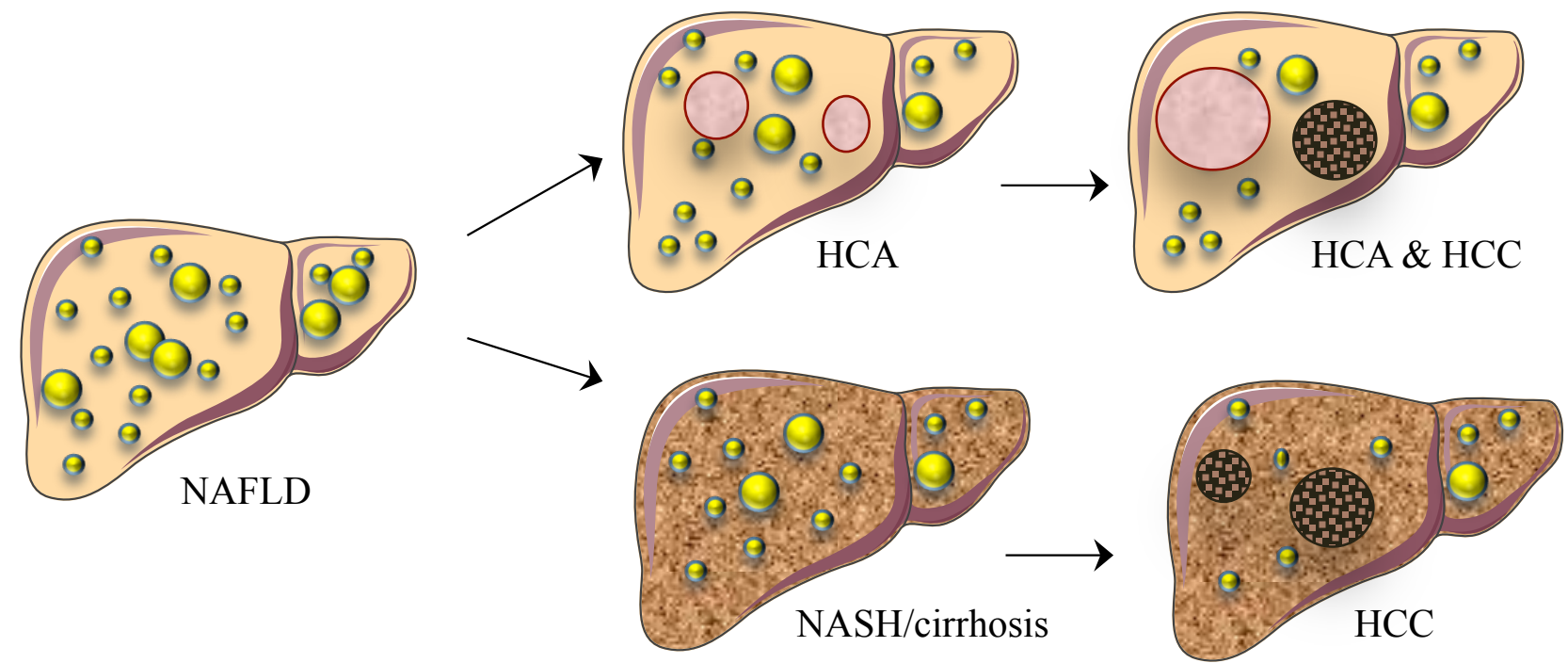

Figure 3 


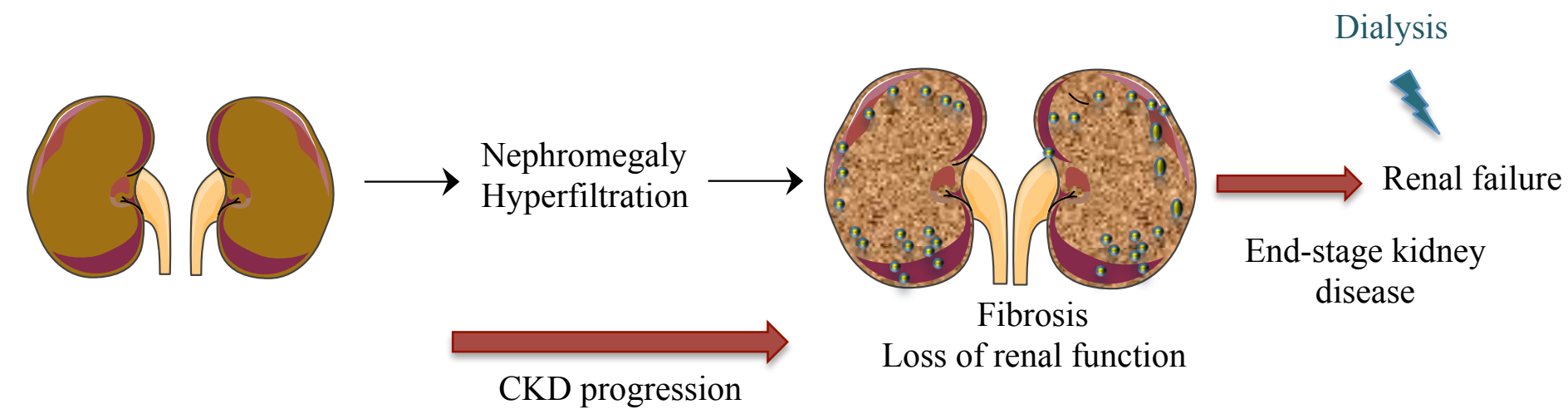

Figure 4 